\title{
SUM OF SQUARES MANIFOLDS: THE EXPRESSIBILITY OF THE LAPLACE-BELTRAMI OPERATOR ON PSEUDO-RIEMANNIAN MANIFOLDS AS A SUM OF SQUARES OF VECTOR FIELDS
}

\author{
WILFRIED H. PAUS
}

To my aunt Ingrid S. Keller

\begin{abstract}
In this paper, we investigate under what circumstances the Laplace-Beltrami operator on a pseudo-Riemannian manifold can be written as a sum of squares of vector fields, as is naturally the case in Euclidean space.

We show that such an expression exists globally on one-dimensional manifolds and can be found at least locally on any analytic pseudo-Riemannian manifold of dimension greater than two. For two-dimensional manifolds this is possible if and only if the manifold is flat.

These results are achieved by formulating the problem as an exterior differential system and applying the Cartan-Kähler theorem to it.
\end{abstract}

\section{INTRODUCTION}

We study an $n$-dimensional real pseudo-Riemannian (sometimes called semiRiemannian) manifold (that is, a pair $(\mathcal{M}, g)$ of a differential manifold $\mathcal{M}$ and its metric $g$ ) and a symmetric nondegenerate $(0,2)$-tensor field on $\mathcal{M}$ of constant index. The central object of our investigation will be the Laplace-Beltrami operator on such a manifold.

1.1. Notation and preliminaries. Arbitrary (open) subsets of a differential manifold $\mathcal{M}$ are denoted by $\mathcal{U}$. The tangent space at $p \in \mathcal{U}$ is always indicated by $T_{p} \mathcal{U}$; similarly we write $T_{p}^{*} \mathcal{U}$ for its dual. We also use the usual notation $\mathfrak{X}(\mathcal{U})$ for the set of vector fields on $\mathcal{U}$ and write $\Omega^{r}(\mathcal{U})$ for the set of differential $r$-forms defined there. The neutral element; in these and other sets are denoted by 0 if the objects being considered are $\mathbb{R}$-valued (e.g., numbers, functions) and by $O$ if the objects have components (e.g., vector fields, matrices, differential forms).

By the letter $\mathcal{V}$, we denote an open subset of the manifold which admits a local coordinate system $\mathbf{x}: \mathcal{V} \longrightarrow \mathbb{R}^{n}: q \mapsto\left(x_{1}(q), \ldots, x_{n}(q)\right)$. On such a set, we use

Received by the editors December 30, 1996.

1991 Mathematics Subject Classification. Primary 58G03; Secondary 58A15, 53C21.

Key words and phrases. Differential geometry, pseudo-Riemannian manifolds, the LaplaceBeltrami operator, exterior differential systems, Cartan-Kähler theory.

This work was made possible through funding from the Australian Department of Employment, Education and Training (OPRS), the Deutscher Akademischer Austauschdienst of Germany, the Australian Research Council Grant "Differential and Integral Operators", and the Deutsche Forschungsgemeinschaft. 
the standard matrix representation of any pseudo-Riemannian metric $g$ on $\mathcal{M}$ with respect to the canonical corresponding vector fields $\left(\frac{\partial}{\partial x_{i}}\right)_{i}$ by writing

$$
G:=\left(g_{i j}\right):=\left(g\left(\frac{\partial}{\partial x_{i}}, \frac{\partial}{\partial x_{j}}\right)\right)
$$

to obtain a matrix-valued mapping $G$ from $\mathcal{V}$ into the set of real invertible symmetric matrices. For the mapping from $\mathcal{V}$ onto the inverse of $G$, we write $G^{-1}=:\left(g^{i j}\right)$, i. e., $\sum_{j=1}^{n} g^{i j} g_{j k}=\delta_{i k}$, the Kronecker delta.

We use $\nabla$ to denote the covariant derivatives on $(\mathcal{M}, g)$. In order to perform covariant differentiation a certain level of regularity is required, so we assume all vector fields and differential forms appearing in this paper to be at least once differentiable. We demand the same of the pseudo-Riemannian metric.

Let us clarify the use of covariant differentiation. For any vector field $X \in \mathfrak{X}(\mathcal{M})$ the operator $\nabla_{X}$ denotes the pseudo-Riemannian connection acting on $\mathfrak{X}(\mathcal{M})$ as usual (see [11], Chap. I, §9). Moreover, we consider $\nabla_{X}$ as a derivation on the Grassmann algebra of alternating differential forms with the pointwise definition

$$
\left(\nabla_{X} \sigma\right)\left(Z_{1}, \ldots, Z_{r}\right):=X\left(\sigma\left(Z_{1}, \ldots, Z_{r}\right)\right)-\sum_{i=1}^{r} \sigma\left(Z_{1}, \ldots, \nabla_{X} Z_{i}, \ldots, Z_{r}\right)
$$

for all $X, Z_{1}, \ldots, Z_{r} \in \mathfrak{X}(\mathcal{M})$ and any $r$-form $\sigma \in \Omega^{r}(\mathcal{M})$ (see [14], Chap. VIII, $\left.§ 1\right)$. In contrast to some references (e.g., [8]), we follow [25] by regarding $\nabla_{X} \sigma$ as an $r$-form when carrying out further covariant differentiation, i.e., for the second-order covariant derivatives we set

$$
\nabla_{X Y}^{2} \sigma=\nabla_{X} \nabla_{Y} \sigma-\nabla_{\nabla_{X} Y} \sigma \quad \text { for all } X, Y \in \mathfrak{X}(\mathcal{M}) \text { and } \sigma \in \Omega^{r}(\mathcal{M})
$$

with

$$
\begin{aligned}
\left(\nabla_{Y} \nabla_{X} \sigma\right)\left(Z_{1}, \ldots, Z_{r}\right):= & Y\left(\left(\nabla_{X} \sigma\right)\left(Z_{1}, \ldots, Z_{r}\right)\right) \\
& -\sum_{i=1}^{r}\left(\nabla_{X} \sigma\right)\left(Z_{1}, \ldots, \nabla_{Y} Z_{j}, \ldots, Z_{r}\right)
\end{aligned}
$$

for all $X, Y, Z_{1}, \ldots, Z_{r} \in \mathfrak{X}(\mathcal{M})$, in conformity with (1.1). This is, however, just a matter of notation.

In the sequel we frequently utilise collections of vector fields $X_{1}, \ldots, X_{n} \in \mathfrak{X}(\mathcal{U})$ defined on some subset $\mathcal{U}$ of an $n$-dimensional differential manifold, which form pointwise bases of the tangent spaces, i. e., where $\left(\left.X_{i}\right|_{p}\right)_{i}$ is a basis of $T_{p} \mathcal{U}$ for every $p$ in $\mathcal{U}$. As is customary, we refer to such a list $\left(X_{i}\right)_{i}$ as a frame. Recall in this connection that a differential manifold is called parallelisable if and only if it admits a frame.

The presence of a pseudo-Riemannian metric on a differential manifold leads to the notion of orthonormality on the tangent spaces. We recall a few definitions and facts in this connection (see, e.g., [17]).

Definition 1.1. Let $\mathcal{U}$ be an open subset of a pseudo-Riemannian manifold $(\mathcal{M}, g)$ of dimension $n$. A list $\left(Y_{i}\right)_{i}$ of $n$ vector fields is, or the vector fields themselves are, called $g$-orthonormal if

$$
g\left(Y_{i}, Y_{j}\right)=\epsilon_{j} \delta_{i j} \text { with } \epsilon_{j}:=g\left(Y_{j}, Y_{j}\right)= \pm 1 \quad \text { for all } i, j=1, \ldots, n .
$$


The $n$-tuple $\left(\epsilon_{1}, \ldots, \epsilon_{n}\right)$ is usually called the signature of $g$ (with respect to $\left.\left(Y_{i}\right)_{i}\right)$. The number of negative signs in a signature is the same for any choice of $g$-orthonormal vector fields, and is referred to as the index of the manifold. In the case of index 0 the pseudo-Riemannian metric $g$ is positive definite and the prefix "pseudo" in all of the above is omitted. It is a well-known fact that one can construct locally a list of $g$-orthonormal vector fields on any pseudo-Riemannian manifold. In the Riemannian case this is even true globally, provided the manifold is parallelisable.

Analogously we call a collection of differential 1-forms $\omega_{1}, \ldots, \omega_{n}$ on an open subset $\mathcal{U}$ of a differential manifold a coframe if the list $\left(\left.\omega_{k}\right|_{p}\right)_{k}$ forms a basis of $T_{p}^{*} \mathcal{U}$ for every $p$ in $\mathcal{U}$. For every frame $\left(X_{i}\right)_{i}$ there exists a unique dual coframe $\left(\omega_{k}\right)_{k}$ which is determined by the relation

$$
\omega_{k}(p)\left(\left.X_{i}\right|_{p}\right)=\delta_{i k} \quad \text { for all } i, k=1, \ldots, n \text { and every } p \text { in } \mathcal{U}
$$

and vice versa. A coframe is called $g$-orthonormal if and only if its dual vector fields have this property.

Remark 1.2. To express the property of $g$-orthonormality of $\left(Y_{i}\right)$ in terms of local coordinates $\left(x_{i}\right)_{i}$ on some open subset $\mathcal{V} \subseteq \mathcal{U}$, write

$$
Y_{i}=\sum_{j=1}^{n} b_{i j} \frac{\partial}{\partial x_{j}} \quad \text { for all } i=1, \ldots, n .
$$

Then we have the following condition for the coefficients $b_{i j}$ of these vector fields:

$$
\epsilon_{j} \delta_{i j}=g\left(Y_{i}, Y_{j}\right)=g\left(\sum_{k=1}^{n} b_{i k} \frac{\partial}{\partial x_{k}}, \sum_{l=1}^{n} b_{j l} \frac{\partial}{\partial x_{l}}\right)=\sum_{k, l=1}^{n} b_{i k} b_{j l} g_{k l} .
$$

Denoting $B:=\left(b_{i j}\right)$, this identity reads, in matrix form,

$$
J=B \cdot G \cdot B^{*} \quad \text { or, equivalently, } \quad G^{-1}=B^{*} \cdot J \cdot B,
$$

where we denote the standard matrix multiplication by $\bullet$. Here $J$ is the signature matrix associated with $\left(Y_{i}\right)_{i}$, i.e., $J:=\left(\epsilon_{j} \delta_{i j}\right)=J^{*}=J^{-1}$, which contains precisely as many minus signs on its main diagonal as the index of the manifold.

1.2. The Laplace-Beltrami operator. We deal with differential operators on a pseudo-Riemannian manifold $(\mathcal{M}, g)$, in particular the Laplace-Beltrami operator

$$
\Delta:=\operatorname{div} \text { grad } .
$$

Here

$$
g(\operatorname{grad} f, X):=X f
$$

and

$$
\operatorname{div} X=\sum_{i=1}^{n} \epsilon_{i} g\left(\nabla_{Y_{i}} X, Y_{i}\right)
$$

in either case for all $X \in \mathfrak{X}(\mathcal{M})$, where $\left(Y_{i}\right)_{i}$ is an arbitrary $g$-orthonormal frame of signature $\left(\epsilon_{1}, \ldots, \epsilon_{n}\right)$ given locally on $\mathcal{M}$. With respect to local coordinates the Laplace-Beltrami operator (Laplacian for short) is given by

$$
\Delta=\frac{1}{\sqrt{|\operatorname{det} G|}} \sum_{j=1}^{n} \frac{\partial}{\partial x_{j}}\left(\sum_{i=1}^{n} g^{i j} \sqrt{|\operatorname{det} G|} \cdot \frac{\partial}{\partial x_{i}}\right) \text {. }
$$


Alternatively, one has

$$
\Delta=\sum_{i, j=1}^{n} g^{i j} \nabla_{\frac{\partial}{\partial x_{i}}}^{2} \frac{\partial}{\partial x_{j}}
$$

with respect to covariant derivatives (cf. [8], §26). Recalling the definition of the Laplacian on $\mathbb{R}^{n}$

$$
\Delta_{\mathbb{R}^{n}}=\sum_{i=1}^{n} \frac{\partial^{2}}{\partial x_{i}^{2}}
$$

we may describe its structure by the following three properties:

(i) $\Delta_{\mathbb{R}^{n}}$ is symmetric on the Lebesgue space $L^{2}\left(\mathbb{R}^{n}\right)$ of squareintegrable real-valued functions on $\mathbb{R}^{n}$ equipped with the usual inner product $L^{2}\left(\mathbb{R}^{n}\right) \times L^{2}\left(\mathbb{R}^{n}\right) \rightarrow \mathbb{R}:(f, g) \mapsto \int_{\mathbb{R}^{n}} f g$,

(ii) $\Delta_{\mathbb{R}^{n}}$ can be written as a sum of squares of linear first-order differential operators, and

(iii) these operators commute.

Hence it is a natural question to ask whether the Laplacian on $\mathcal{M}$ (and thus on any (open) subset $\mathcal{U} \subseteq \mathcal{M}$ ) has similar features when we replace $\mathbb{R}^{n}$ by $\mathcal{M}$ in the above. The first of these properties follows from the definition (1.3) of $\Delta$ as a "div grad" operator. Indeed, as in $\mathbb{R}^{n}$, one has Green's formula, which implies that the Laplace-Beltrami operator is symmetric (see [4] Theorem 3.18). Note that in the Riemannian case it is also negative.

When investigating the property (1.7) (ii), a look at the Laplacian on fourdimensional Minkowski space $\mathbb{M}$

$$
\Delta_{\mathbb{M}}:=-\frac{\partial^{2}}{\partial t^{2}}+\sum_{i=1}^{3} \frac{\partial^{2}}{\partial x_{i}^{2}}
$$

suggests that the signature of the manifold has to be taken into consideration when looking for such an expression. One would therefore seek a list of $n$ vector fields $Y_{i}$ on $\mathcal{M}$ such that

$$
\Delta=\sum_{i=1}^{n} \pm Y_{i} \circ Y_{i}=\sum_{i=1}^{n} \pm Y_{i}^{2}
$$

with the signs chosen in accordance with the given metric. Considering the functions on $\mathcal{M}$ as 0 -forms, this is, in view of (1.2), the same as demanding that the $Y_{i}$ satisfy

$$
\Delta=\sum_{i=1}^{n} \pm \nabla_{Y_{i}} \nabla_{Y_{i}},
$$

since $\nabla_{Y_{i}} f=Y_{i}(f)$ for all $f \in \mathrm{C}^{1}(\mathcal{M})$. There appears to be some confusion about the existence of such a representation for $\Delta$. Our goal in this paper is to give a definite answer to this question. Property (1.7) (iii) of the Laplacian, i. e., the possibility that the $Y_{i}$ in (1.8) commute, will be treated along the way. In our investigations we make the following distinction, which will be justified later.

Definition 1.3. Let $(\mathcal{M}, g)$ be a pseudo-Riemannian manifold of index $\nu$ and dimension $n$. 
We say that $(\mathcal{M}, g)$ is a local sum of squares manifold if for every point $p$ in $\mathcal{M}$ there exist a neighbourhood $\mathcal{U}(p) \subseteq \mathcal{M}$ and a list of $n$ vector fields $\left(Y_{i}\right)_{i}$ defined on $\mathcal{U}$, such that the Laplacian there can be written in the form

$$
\Delta=-\sum_{i=1}^{\nu} Y_{i}^{2}+\sum_{i=\nu+1}^{n} Y_{i}^{2} \quad \text { everywhere in } \mathcal{U} .
$$

If such vector fields can be found globally on $\mathcal{M},(\mathcal{M}, g)$ is referred to as a global sum of squares manifold.

Naturally, the order of the signs in (1.9) is just a matter of convention, since one can always renumber the indices of the vector fields involved. For convenience we assign the corresponding lists of vector fields a specific name.

Definition 1.4. Let $\mathcal{U}$ be an open subset of a pseudo-Riemannian manifold. A list of vector fields $\left(Y_{i}\right)_{i}$ satisfying the identity (1.9) everywhere in $\mathcal{U}$ is called a list of sum of squares vector fields (on $\mathcal{U}$ ).

The paper is organised as follows. In the remainder of this section, we treat the two trivial cases with respect to the sum of squares property, that is, onedimensional and flat manifolds. In Section 2 we give a characterisation of sum of squares manifolds in the local as well as in the global sense. In particular, we give a formulation of the sum of squares problem in the language of differential forms. In Section 3, we use this formulation and the Cartan-Kähler theorem to give answers to the local sum of squares problem. These are definite at least in the analytic category. Finally, in Section 4, we state some applications of our results and give a short survey of open and related questions.

1.3. One-dimensional manifolds. One-dimensional manifolds are always global sum of squares manifolds. To see this, note that in the case where $n=1$, we may renounce the use of indices and simply write $x$ for the local coordinate and $g:=g\left(\frac{d}{d x}, \frac{d}{d x}\right)$. Hence $G=(g(p)), G^{-1}=\left(\frac{1}{g(p)}\right)$ and $\operatorname{det} G=g \neq 0$. Locally the Laplacian of a pseudo-Riemannian manifold $(\mathcal{M}, g)$ according to (1.6) then takes the form

$$
\Delta=\frac{1}{\sqrt{|g|}} \frac{d}{d x}\left(\frac{\sqrt{|g|}}{g} \frac{d}{d x}\right)=(-1)^{\nu} \frac{1}{\sqrt{|g|}} \frac{d}{d x}\left(\frac{1}{\sqrt{|g|}} \frac{d}{d x}\right)
$$

this is obviously the square of either one of the vector fields $\pm \frac{1}{\sqrt{|g|}} \frac{d}{d x}$, in the sense of (1.9). Since we can do this for any chart of $\mathcal{M}$, this manifold has the local sum of squares property. Moreover, fixing the sign and successively considering the corresponding local vector field for every chart of a given atlas for $\mathcal{M}$ yields a globally well-defined vector field with the sum of squares property.

1.4. Flat manifolds. Flat manifolds are always local sum of squares manifolds. This is because on a flat pseudo-Riemannian manifold one can always find local coordinates $\left(x_{i}\right)_{i}$ such that $G$ has constant components with respect to these ([15], Theorem 7.5.3). Since the scalar product space $\left(\mathbb{R}^{n},\langle\cdot, G \cdot\rangle\right)$ always admits an orthonormal basis, we may decompose this fixed symmetric matrix as in Remark 1.2 by $G^{-1}=B^{*} \cdot J \cdot B$ with some $B \in \mathrm{GL}(n, \mathbb{R})$ and a signature matrix $J=\left(\epsilon_{j} \delta_{i j}\right)$. 
Since all the terms involving $g$ in (1.6) are constant, the Laplacian on the flat manifold may then be written in the form

$$
\begin{aligned}
\Delta & =\frac{1}{\sqrt{|\operatorname{det} G|}} \sum_{i, j=1}^{n} \frac{\partial}{\partial x_{j}}\left(g^{i j} \sqrt{|\operatorname{det} G|} \cdot \frac{\partial}{\partial x_{i}}\right)=\sum_{i, j=1}^{n} \frac{\partial}{\partial x_{j}}\left(\sum_{k=1}^{n} \epsilon_{k} b_{k i} b_{k j} \cdot \frac{\partial}{\partial x_{i}}\right) \\
& =\sum_{k=1}^{n} \epsilon_{k}\left(\sum_{j=1}^{n} b_{k j} \frac{\partial}{\partial x_{j}}\right)\left(\sum_{i=1}^{n} b_{k i} \cdot \frac{\partial}{\partial x_{i}}\right),
\end{aligned}
$$

i. e., it is locally given by the sum of squares of the vector fields

$$
\sum_{i=1}^{n} b_{k i} \cdot \frac{\partial}{\partial x_{i}} \quad(k=1, \ldots, n) .
$$

We will see in the next section that flat manifolds are not necessarily global sum of squares manifolds.

\section{ACKNOWLEDGEMENTS}

This paper develops the main result of my Ph.D. dissertation [18] which I wrote under the guidance of Professor Michael Cowling. I thank him very much for all his support. Moreover, many thanks to Ian Lisle and Peter Vassiliou for many stimulating discussions, and in particular to the editor, Professor Robert Bryant, whose suggestions provided the basis for the proof of the result given below in the Cartan-Kähler setting.

\section{Characterisations of SUm of Squares manifolds}

Our goal in this section is to derive some special properties of local and global sum of squares manifolds.

2.1. Parallelisability of sum of squares manifolds. As a first characterisation, note that any list of sum of squares vector fields has to be a $g$-orthonormal frame.

Lemma 2.1. Let $(\mathcal{M}, g)$ be a pseudo-Riemannian manifold of index $\nu$ and dimension $n$, and let $\mathcal{U} \subseteq \mathcal{M}$ be open. If the Laplacian on $\mathcal{U}$ can be written in the form (1.9) with $Y_{i} \in \mathfrak{X}(\mathcal{U})$, then the list $\left(Y_{i}\right)_{i}$ is g-orthonormal in $\mathcal{U}$.

Proof. Consider the representation (1.6) for the Laplacian in local coordinates and compare the coefficients of its second-order terms with those of the sum of squares expression (1.9) with the vector fields $Y_{i}$, locally written as $Y_{i}=: \sum_{j=1}^{n} b_{i j} \frac{\partial}{\partial x_{j}}$ for $i=1, \ldots, n$. With respect to the standard signature

$$
\left(\epsilon_{i}\right)_{i}:=(-1, \ldots,-1,1, \ldots, 1),
$$

we obtain the condition

$$
\sum_{i=1}^{n} \epsilon_{i} b_{i j} b_{i k}=g^{j k} \quad \text { for all } j, k=1, \ldots, n,
$$

and thus the $g$-orthonormality of the $Y_{i}$ according to Remark 1.2. 
Remark 2.2. The assertion of the lemma implies that a list of at least $n$ vector fields on an open subset of a manifold of this dimension is needed to form the Laplacian there as the sum of their squares. Any lower number of vector fields could not satisfy the orthonormality condition (2.2), because the rank of $B=\left(b_{i j}\right)$ has to be equal to $n$ for $G^{-1}$ to be regular. As stated in Section 1.2, we restrict our considerations to this optimal case, i.e., to investigating the existence of exactly $n$ sum of squares vector fields.

Considering the case where $\mathcal{U}=\mathcal{M}$, we obtain a strong necessary condition for global sum of squares manifolds.

Corollary 2.3. Every global sum of squares manifold is parallelisable.

This yields the first examples of Riemannian manifolds that have no global sum of squares expression for their Laplacian. Consider, for instance, the $n$-dimensional unit spheres for $n \neq 1,3,7$ or the Möbius strip (which even admits a flat connection).

Open parallelisable subsets $\mathcal{U}$ of a differentiable manifold $\mathcal{M}$ can be treated in a similar way to coordinate subsets. This is because one can use a frame $\left(X_{i}\right)_{i}$ on $\mathcal{U}$ as one would use the canonical basis $\left(\frac{\partial}{\partial x_{i}}\right)_{i}$ of $\mathfrak{X}(\mathcal{U})$, if local coordinates $\left(x_{i}\right)_{i}$ were given. The main difference between such an arbitrary list and the special case where $X_{i}=\frac{\partial}{\partial x_{i}}$ is that two distinct such vector fields do not necessarily commute. In particular, commutativity and $g$-orthonormality are two properties of vector fields that usually exclude each other.

Lemma 2.4. Let $\mathcal{U}$ be an open subset of a pseudo-Riemannian manifold $(\mathcal{M}, g)$ with a list $\left(Y_{i}\right)_{i}$ of commuting and $g$-orthonormal vector fields defined on it. Then $(\mathcal{U}, g)$ is flat.

Proof. Pick a point $p$ arbitrarily in $\mathcal{U}$. Since the $Y_{i}$ commute, the Frobenius theorem (cf. [6], Theorem 4.3.1) states that there exists a neighbourhood $\mathcal{V} \subseteq \mathcal{U}$ of $p$ admitting a local coordinate system $\left(y_{i}\right)_{i}$ where

$$
Y_{i}=\frac{\partial}{\partial y_{i}} \quad \text { for all } i=1, \ldots, n
$$

Moreover, since the $Y_{i}$ are $g$-orthonormal, we have that

$$
g_{i j}=g\left(\frac{\partial}{\partial y_{i}}, \frac{\partial}{\partial y_{j}}\right)=g\left(Y_{i}, Y_{j}\right)=\epsilon_{j} \delta_{i j} \quad \text { for all } i, j=1, \ldots, n \text { everywhere in } \mathcal{V}
$$

with respect to these coordinates. Hence the coefficients of the pseudo-Riemannian metric are locally constant here. Thus $(\mathcal{V}, g)$ has to be flat. Since $p$ was chosen arbitrarily, this property has to hold in all of $\mathcal{U}$.

Sum of squares vector fields are always $g$-orthonormal, and so we obtain an answer to the question of when the property (1.7) (iii) holds on pseudo-Riemannian manifolds.

Corollary 2.5. If a pseudo-Riemannian manifold admits a list of sum of squares vector fields which commute, then it is flat.

In Section 1.4 we have already shown the converse, i. e., that flat manifolds are always local sum of squares manifolds and that the corresponding vector fields can be chosen to commute. 
2.2. Sum of squares criteria. We refine the necessary condition given above to one which is also sufficient. For this, observe that the Laplacian on an open subset of a pseudo-Riemannian manifold always can be written in a special form when employing $g$-orthonormal frames. The first of the following assertions is well-known at least for specific Riemannian manifolds.

Lemma 2.6. Let $\mathcal{U}$ be an arbitrary open subset of an n-dimensional pseudo-Riemannian manifold. Then, for any g-orthonormal frame $\left(Y_{i}\right)_{i}$ on $\mathcal{U}$ with $\epsilon_{i}=$ $g\left(Y_{i}, Y_{i}\right)$, one has

$$
\begin{aligned}
& \text { (i) } \Delta=\sum_{i=1}^{n}\left(\epsilon_{i} Y_{i}^{2}+\left(\operatorname{div} Y_{i}\right) \cdot Y_{i}\right), \\
& \text { (ii) } \Delta=\sum_{i=1}^{n} \epsilon_{i}\left(Y_{i}^{2}-\nabla_{Y_{i}} Y_{i}\right),
\end{aligned}
$$

in both cases everywhere in $\mathcal{U}$.

Proof. Using the identity (1.5) we calculate, for an arbitrary function $f \in C^{2}(\mathcal{U})$, that

$$
\begin{aligned}
\Delta f & =\sum_{i=1}^{n} \epsilon_{i} g\left(\nabla_{Y_{i}} \operatorname{grad} f, Y_{i}\right) \\
& =\sum_{i=1}^{n} \epsilon_{i}\left(Y_{i} g\left(\operatorname{grad} f, Y_{i}\right)-g\left(\operatorname{grad} f, \nabla_{Y_{i}} Y_{i}\right)\right) \\
& =\sum_{i=1}^{n} \epsilon_{i}\left(Y_{i} \circ Y_{i} f-\nabla_{Y_{i}} Y_{i} f\right),
\end{aligned}
$$

because of the symmetry of the pseudo-Riemannian connection and (1.4). This proves (ii). Similar arguments yield

$$
\begin{aligned}
\sum_{i=1}^{n} \epsilon_{i} \nabla_{Y_{i}} Y_{i} & =\sum_{i, j=1}^{n} \epsilon_{i} g\left(\nabla_{Y_{i}} Y_{i}, Y_{j}\right) Y_{j} \\
& =\sum_{i, j=1}^{n} \epsilon_{i} Y_{i} g\left(Y_{i}, Y_{j}\right) Y_{j}-\sum_{i, j=1}^{n} \epsilon_{i} g\left(\nabla_{Y_{i}} Y_{j}, Y_{i}\right) Y_{j} \\
& =-\sum_{j=1}^{n}\left(\operatorname{div} Y_{j}\right) \cdot Y_{j}
\end{aligned}
$$

by virtue of the orthonormal decomposition theorem and since $Y_{i} g\left(Y_{j}, Y_{k}\right)=Y_{i} \delta_{j k}$ vanishes for all $i, j, k=1, \ldots, n$. Inserting (2.3) into identity (ii) now gives (i).

The identities just established can be used to characterise the sum of squares property on $\mathcal{U}$.

Proposition 2.7. Let $\mathcal{U}$ be an arbitrary open subset of a pseudo-Riemannian manifold $(\mathcal{M}, g)$ of index $\nu$ and dimension $n$, and let $\left(Y_{1}, \ldots, Y_{n}\right)$ be a list of (differentiable) vector fields on $\mathcal{U}$. Then the following assertions are equivalent: 
(i) $\left(Y_{1}, \ldots, Y_{n}\right)$ is a list of sum of squares vector fields, i. e., the Laplacian $\Delta$ on $\mathcal{U}$ is of the form

$$
\Delta=-\sum_{i=1}^{\nu} Y_{i}^{2}+\sum_{i=\nu+1}^{n} Y_{i}^{2} .
$$

(ii) The vector fields $Y_{1}, \ldots, Y_{n}$ are g-orthonormal with signature (2.1) and divergence-free on $\mathcal{U}, i$. e.,

$$
\operatorname{div} Y_{i}=0 \quad \text { for all } i=1, \ldots, n .
$$

(iii) The vector fields $Y_{1}, \ldots, Y_{n}$ are $g$-orthonormal with signature (2.1) and satisfy the identity

$$
\sum_{i=1}^{n} \epsilon_{i} \nabla_{Y_{i}} Y_{i}=-\sum_{i=1}^{\nu} \nabla_{Y_{i}} Y_{i}+\sum_{i=\nu+1}^{n} \nabla_{Y_{i}} Y_{i}=O \quad \text { everywhere in } \mathcal{U}
$$

Proof. We show first that (i) implies (ii). If $\Delta=-\sum_{i=1}^{\nu} Y_{i}^{2}+\sum_{i=\nu+1}^{n} Y_{i}^{2}$, the $Y_{i}$ have to be $g$-orthonormal with the given signature by Lemma 2.1. Hence, Lemma 2.6 (i) implies that

$$
O=\Delta-\sum_{i=1}^{n} \epsilon_{i} Y_{i}^{2}=\sum_{i=1}^{n}\left(\operatorname{div} Y_{i}\right) \cdot Y_{i}
$$

Since the list $\left(Y_{i}\right)_{i}$ is a frame, all factors in this linear combination of the $Y_{i}$ have to be zero, i. e.,

$$
\operatorname{div} Y_{i}=0 \quad \text { for all } i=1, \ldots, n .
$$

That (ii) implies (iii) is a direct consequence of (2.3), and (iii) implies (i) by Lemma 2.6 (ii).

In order to formulate this result with respect to differential forms, consider the dual forms $\left(\omega_{k}\right)_{k}$ of a list of sum of squares vector fields. By the proposition these $\omega_{k}$ have to form a $g$-orthonormal coframe.

To consider the effect of the divergence-freeness of each $Y_{i}$ on $\left(\omega_{k}\right)_{k}$, recall that, for the forms dual to a frame $\left(Y_{i}\right)_{i}$, one has that

$$
\operatorname{div} Y_{i}=* d * \omega_{i}=-\delta \omega_{i} \quad \text { for all } i=1, \ldots, n .
$$

Here, $d$ and $*$ denote the exterior derivative and the Hodge operator, respectively. The letter $\delta$ denotes the codifferential, i. e., the operator adjoint to $d$ with respect to the canonical inner product on the Grassmann algebra on $\mathcal{U}$, given by

$$
\delta=(-1)^{n(r+1)+1+\nu} * d *
$$

when acting on $r$-forms. See, for instance, [1] for details. Applying the above, it follows that a list of $g$-orthonormal vector fields $\left(Y_{i}\right)_{i}$ has the sum of squares property on $\mathcal{U}$ if and only if for the dual forms $\left(\omega_{k}\right)_{k}$, the equations

$$
\delta \omega_{k}=O \quad \text { for all } k=1, \ldots, n
$$

all hold, i. e., they have vanishing adjoint exterior derivative.

To obtain an alternative formulation of the above, recall that a volume form on an open subset $\mathcal{U}$ of an $n$-dimensional pseudo-Riemannian manifold $(\mathcal{M}, g)$ is a smooth $n$-form $d V$ such that

$$
d V\left(Y_{1}, \ldots, Y_{n}\right) \equiv \pm 1 \text { in } \mathcal{U} \text { for every } g \text {-orthonormal frame }\left(Y_{i}\right)_{i} \text { on } \mathcal{U} \text {. }
$$


Clearly, $\omega_{1} \wedge \cdots \wedge \omega_{n}$ defines a volume form for any $g$-orthonormal coframe $\left(\omega_{k}\right)_{k}$. Then, by definition of the Hodge operator, we have for any $k=1, \ldots, n$ that

$$
* \omega_{k}=\epsilon_{k}(-1)^{k-1} \omega_{1} \wedge \cdots \wedge \widehat{\omega_{k}} \wedge \cdots \wedge \omega_{n},
$$

where we use the symbol $\widehat{-}$ to denote omission. Thus (2.4) may also be written as

$$
O=* O=* \delta \omega_{k}=-* * d * \omega_{k}=\epsilon_{k}(-1)^{n-k+1+\nu} d\left(\omega_{1} \wedge \cdots \wedge \widehat{\omega_{k}} \wedge \cdots \wedge \omega_{n}\right)
$$

for all $k=1, \ldots, n$, because

$$
* *=(-1)^{r(n-r)+\nu}
$$

on $r$-forms. Hence, a $g$-orthonormal coframe $\left(\omega_{k}\right)_{k}$ is dual to a list of sum of squares vector fields if and only if all the $(n-1)$-forms

$$
\omega_{1} \wedge \cdots \wedge \widehat{\omega_{k}} \wedge \cdots \wedge \omega_{n}
$$

are closed. Summarising, Proposition 2.7 may also be stated as follows.

Proposition 2.8. Let $\mathcal{U}$ be an open subset of an n-dimensional pseudo-Riemannian manifold $(\mathcal{M}, g)$ of index $\nu$. Then the following assertions are equivalent:

(i) The Laplacian on $\mathcal{U}$ can be expressed as a sum of squares of vector fields, i. e., there exist $Y_{1}, \ldots, Y_{n} \in \mathfrak{X}(\mathcal{U})$ such that

$$
\Delta=-\sum_{i=1}^{\nu} Y_{i}^{2}+\sum_{i=\nu+1}^{n} Y_{i}^{2} .
$$

(ii) There exists a g-orthonormal coframe $\left(\omega_{1}, \ldots, \omega_{n}\right) \subseteq \Omega^{1}(\mathcal{U})$ of signature $(2.1)$ where each element has vanishing codifferential.

(iii) There exists a g-orthonormal coframe $\left(\omega_{1}, \ldots, \omega_{n}\right) \subseteq \Omega^{1}(\mathcal{U})$ of signature $(2.1)$ where each element is co-closed, $i$. e., $d\left(\omega_{1} \wedge \cdots \wedge \widehat{\omega_{k}} \wedge \cdots \wedge \omega_{n}\right)=O$ for all $k=1, \ldots, n$.

The $\omega_{k}$ in (ii) can be chosen to be dual with respect to the $Y_{i}$ in (i), and vice versa.

Remark 2.9. Observe that this is true in particular when each of the $\omega_{k}$ has this property, i. e., $d \omega_{k}=O$ for all $k=1, \ldots, n$. However, since we demand that the $\omega_{k}$ also form a $g$-orthonormal coframe, it is unlikely that such a list of Pfaffian forms can be found. As a matter of fact, one can achieve this only on flat manifolds by Corollary 2.5.

\section{LOCAL EXISTENCE OF SUM OF SQUARES VECTOR FIELDS}

In [18], the author related the question of the existence of sum of squares vector fields to the solvability of a degenerate system of first-order quasilinear partial differential equations. Because of the degeneracy of this system the well-known Cauchy-Kowalevskaya theorem could not be used for an existence proof if $n \geq 3$. It was necessary to utilise a more general power series method, the Riquier-Janet theory (see, e. g., [19]) in a very technical manner. Here we prove the same results as in [18] by means of the calculus of exterior differential systems and in particular the Cartan-Kähler theorem. These proofs, which are based on a suggestion of Professor Robert Bryant, are shorter and more transparent than the original ones.

An exterior differential system is a system of equations on a manifold defined by equating to zero a number of differential forms. The theory of these systems provides an alternative way of solving partial differential equations. This is because 
any system of partial differential equations can be written as an exterior differential system and vice versa. The advantages of investigating the latter lie in their coordinate-freeness as well as in the possibility of obtaining additional geometric information (for instance, on symmetries) on the system. See, for instance, [3] for a detailed exposition.

The theory of solving exterior differential systems focuses on the study of their integral manifolds, i. e., submanifolds where all equations of the system are satisfied. The main existence result here is the Cartan-Kähler theorem. This theorem is a coordinate-free, geometric generalisation of the Cauchy-Kowalevskaya theorem; in this sense it corresponds to the Riquier-Janet theory. In particular, it also requires analytic data and yields merely local existence.

In order to apply the Cartan-Kähler theory to the sum of squares problem on a pseudo-Riemannian manifold $(\mathcal{M}, g)$ of index $\nu$ and dimension $n$, we require an exterior differential system that is equivalent to the local sum of squares property of this manifold. Proposition 2.8 provides the first step in this direction. We seek a $g$-orthonormal coframe $\left(\omega_{k}\right)_{k}$ in which each element is co-closed, i. e.,

$$
d\left(\omega_{1} \wedge \cdots \wedge \widehat{\omega_{k}} \wedge \cdots \wedge \omega_{n}\right)=O \text { for all } k=1, \ldots, n .
$$

A more convenient way for the application of the Cartan-Kähler theory is to formulate our problem on the $g$-orthonormal coframe bundle of $(\mathcal{M}, g)$.

3.1. Sections of the $g$-orthonormal coframe bundle. Recall that the $g$-orthonormal coframe bundle of $\mathcal{M}$ is defined by

$$
F(\mathcal{M}):=\bigcup_{p \in \mathcal{M}}\left\{\left(\omega_{i}(p)\right)_{i} \in\left(T_{p}^{*} \mathcal{M}\right)^{n} \mid \begin{array}{l}
\text { dual to a } g_{p} \text {-orthonormal basis of } T_{p} \mathcal{M} \text { of } \\
\text { signature }(2.1)
\end{array}\right\},
$$

i. e., the fibre bundle with base space $\mathcal{M}$ and the set of $g$-orthonormal coframes on $\mathcal{M}$ as fibres. The structure group of $F(\mathcal{M})$ is given by the group of matrices that leave the signature matrix $J$ of the manifold (as defined in Remark 1.2) invariant. This set is commonly denoted by

$$
\mathrm{O}(\nu, n-\nu):=\left\{S \in \mathrm{GL}(n, \mathbb{R}) \mid S^{*} \cdot J \cdot S=J\right\}
$$

and referred to as the semiorthogonal group (of index $\nu$ ) (for details see [17], Chap. 9). $\mathrm{O}(\nu, n-\nu)$ is a Lie group of dimension

$$
N:=\frac{n(n-1)}{2},
$$

so that $F(\mathcal{M})$ defines an $(n+N)$-dimensional differential manifold. A differentiable section of this bundle, i. e., a differentiable mapping

$$
\sigma: \mathcal{M} \rightarrow F(\mathcal{M}): p \mapsto\left(p,\left(\sigma_{i}(p)\right)_{i}\right),
$$

now represents a $g$-orthonormal coframe on the manifold. On $F(\mathcal{M})$, one has canonical 1-forms $\eta_{1}, \ldots, \eta_{n}$ that project onto the components of the fibres. These naturally satisfy the relation

$$
\sigma^{*}\left(\eta_{i}\right):=\eta_{i}(\sigma)=\sigma_{i}
$$

for the pullback of any section $\sigma$. The elements of any $g$-orthonormal coframe $\left(\sigma_{i}\right)$ are independent on $\mathcal{M}$, i. e., $\sigma_{1} \wedge \cdots \wedge \sigma_{n} \neq O$. With respect to the $\eta_{i}$ this 
independence condition reads

$$
\eta_{1} \wedge \cdots \wedge \eta_{n} \neq O
$$

Associated with the $\eta_{i}$ there are also the canonical Levi-Civita connection 1forms $\theta_{i j}=-\theta_{j i}$ on $F(\mathcal{M})$, which satisfy the first Cartan structure equations

$$
d \eta_{i}=\sum_{j=1}^{n} \epsilon_{j} \eta_{j} \wedge \theta_{i j} \quad \text { for all } i=1, \ldots, n .
$$

Note that the set $\left\{\eta_{i}\right\}_{i=1, \ldots, n} \cup\left\{\theta_{i j}\right\}_{1 \leq i<j \leq n}$ defines a coframe on $F(\mathcal{M})$ (see [3], Chap. III, Ex. 3.2). Under a section $\sigma$, the $\theta_{i j}$ pull down to be the usual Levi-Civita connection 1 -forms relative to the $g$-orthonormal coframe that $\sigma$ represents, for the pullback commutes with both the differential and the wedge product. Furthermore, we have the second Cartan structure equations, which relate the connection 1-forms to curvature by

$$
d \theta_{i j}=-\sum_{k=1}^{n} \epsilon_{k} \theta_{i k} \wedge \theta_{k j}+\frac{1}{2} \sum_{k, l=1}^{n} \epsilon_{k} \epsilon_{l} R_{i j k l} \eta_{k} \wedge \eta_{l} \quad \text { for all } i, j=1, \ldots, n
$$

Here, as usual, $R_{i j k l}:=g\left(R\left(Y_{i}, Y_{j}\right) Y_{k}, Y_{l}\right)$ with respect to the vector fields $Y_{i}$ dual to the $g$-orthonormal coframe represented by $\left(\eta_{i}\right)_{i}$. For details on the above see [24], Chaps. 1-2.

We can use the above to reformulate our objective. For this, let

$$
H_{i}:=\epsilon_{i}(-1)^{i-1} \eta_{1} \wedge \cdots \wedge \widehat{\eta}_{i} \wedge \cdots \wedge \eta_{n} \quad \text { for all } i=1, \ldots, n
$$

with the $\eta_{i}$ as in (3.2). Observe the relation with (2.5). Then, by (3.1), a section $\sigma$ of $F(\mathcal{M})$ locally represents a co-closed $g$-orthonormal coframe on $\mathcal{M}$ if and only if

$$
O=d \sigma^{*}\left(H_{i}\right)=\sigma^{*}\left(d H_{i}\right) \text { for all } i=1, \ldots, n
$$

and the independence condition (3.3) holds. In other words, $\sigma(\mathcal{M}) \subset F(\mathcal{M})$ must be a transverse (i.e., with independence condition (3.3)) (n-dimensional) integral manifold of the exterior ideal that is generated by the $n$ differential $n$-forms $d H_{i}$,

$$
\mathcal{I}:=\left\{\sum_{j=1}^{n} \omega_{j} \wedge d H_{j} \mid \omega_{j} \in \Omega(F(\mathcal{M})) \text { of constant degree }\right\} .
$$

For a further simplification of this equivalent formulation of the sum of squares problem, for $n \geq 2$ we define

$$
H_{i j}:=(-1)^{i+j-1} \eta_{1} \wedge \cdots \wedge \widehat{\eta}_{i} \wedge \cdots \wedge \widehat{\eta}_{j} \wedge \cdots \wedge \eta_{n}=:-H_{j i} \quad \text { for all } 1 \leq i<j \leq n
$$

and $H_{i i}:=O$ for all $i=1, \ldots, n$. We have chosen the sign such that

$$
\eta_{i} \wedge \eta_{j} \wedge H_{i j}=\eta_{1} \wedge \cdots \wedge \eta_{n}
$$

With these, we find that

$$
\begin{aligned}
d H_{i} & =\epsilon_{i}(-1)^{i-1} d\left(\eta_{1} \wedge \cdots \wedge \widehat{\eta}_{i} \wedge \cdots \wedge \eta_{n}\right) \\
& =-\epsilon_{i} \sum_{1 \leq j<i} d \eta_{j} \wedge H_{j i}+\epsilon_{i} \sum_{i<j \leq n} d \eta_{j} \wedge H_{i j} \\
& =\epsilon_{i} \sum_{j=1}^{n} d \eta_{j} \wedge H_{i j} .
\end{aligned}
$$


After inserting (3.4), this reads

$$
d H_{i}=\epsilon_{i} \sum_{j, k=1}^{n} \epsilon_{k} \theta_{j k} \wedge \eta_{k} \wedge H_{i j}=\sum_{j=1}^{n}(-1)^{j} \theta_{i j} \wedge \eta_{1} \wedge \cdots \wedge \widehat{\eta}_{j} \wedge \cdots \wedge \eta_{n},
$$

since

$$
\eta_{k} \wedge H_{i j}=\left\{\begin{array}{cl}
(-1)^{j} \eta_{1} \wedge \cdots \wedge \widehat{\eta_{j}} \wedge \cdots \wedge \eta_{n} & \text { if } k=i, \\
(-1)^{i-1} \eta_{1} \wedge \cdots \wedge \widehat{\eta}_{i} \wedge \cdots \wedge \eta_{n} & \text { if } k=j, \\
O & \text { otherwise. }
\end{array}\right.
$$

On the other hand, consider the $(n-1)$-form

$$
\Psi:=\sum_{1 \leq j<k \leq n} \theta_{j k} \wedge H_{j k}
$$

on $F(\mathcal{M})$. It has the property that, for all $i=1, \ldots, n$,

$$
\eta_{i} \wedge \Psi=-\sum_{1 \leq j<k \leq n} \theta_{j k} \wedge \eta_{i} \wedge H_{j k}=\sum_{k=1}^{n}(-1)^{k} \theta_{i k} \wedge \eta_{1} \wedge \cdots \wedge \widehat{\eta_{k}} \wedge \cdots \wedge \eta_{n}
$$

where we have again used (3.7). Consequently,

$$
d H_{i}=\eta_{i} \wedge \Psi \quad \text { for all } i=1, \ldots, n .
$$

Since we require the $\eta_{i}$ to be independent, any integral manifold of $\mathcal{I}$ is also an integral manifold of the exterior ideal generated by merely $\Psi$ (and, of course, vice versa). We may thus restrict our search to the transverse integral manifolds of the latter. Because any integral manifold of an exterior ideal is also an integral manifold of its closure, we further have to include the $n$-form $d \Psi$ in our considerations. A closed exterior ideal is commonly called a differential ideal (see [16], Def. 13.13), so that we have the following.

Proposition 3.1. A pseudo-Riemannian manifold $\mathcal{M}$ is a local sum of squares manifold if and only if its g-orthonormal coframe bundle has an n-dimensional transverse integral manifold of the differential ideal generated by $\Psi$ and $d \Psi$.

In order to establish the existence of sum of squares vector fields we require an explicit expression for $d \Psi$. We first carry out this calculation. In view of the skew-symmetry of $\theta_{i j}$ and $H_{i j}$ we have that

$$
d \Psi=\frac{1}{2} \sum_{i, j=1}^{n}\left(d \theta_{i j} \wedge H_{i j}-\theta_{i j} \wedge d H_{i j}\right) .
$$

The second Cartan structure equation (3.5) yields for the first term the following:

$$
\begin{aligned}
d \theta_{i j} \wedge H_{i j} & =-\sum_{k=1}^{n} \epsilon_{k} \theta_{i k} \wedge \theta_{k j} \wedge H_{i j}+\frac{1}{2} \sum_{k, l=1}^{n} \epsilon_{k} \epsilon_{l} R_{i j k l} \eta_{k} \wedge \eta_{l} \wedge H_{i j} \\
& =\sum_{k=1}^{n} \epsilon_{k} \theta_{k i} \wedge \theta_{k j} \wedge H_{i j}+\epsilon_{i} \epsilon_{j} R_{i j i j} \eta_{1} \wedge \cdots \wedge \eta_{n}
\end{aligned}
$$

for all $i, j=1, \ldots, n$. Therefore, after changing indices, we obtain that

$$
\sum_{i, j=1}^{n} d \theta_{i j} \wedge H_{i j}=\sum_{i, j, k=1}^{n} \epsilon_{i} \theta_{i j} \wedge \theta_{i k} \wedge H_{j k}+\sum_{i, j=1}^{n} \epsilon_{i} \epsilon_{j} R_{i j i j} \eta_{1} \wedge \cdots \wedge \eta_{n} .
$$


Observe that the factor in front of the second term is precisely $n(n-1) K$ with $K$ the scalar curvature of $(\mathcal{M}, g)$ (see [17], Chap. 3, Def. 53). Moreover, a straightforward calculation shows that

$$
d H_{i j}=\sum_{k=1}^{n}\left(\epsilon_{i} \theta_{i k} \wedge H_{j k}-\epsilon_{j} \theta_{j k} \wedge H_{i k}\right) \quad \text { for all } i, j=1, \ldots, n
$$

Consequently, we have that

$$
\begin{aligned}
\sum_{i, j=1}^{n} \theta_{i j} \wedge d H_{i j} & =\sum_{i, j, k=1}^{n}\left(\epsilon_{i} \theta_{i j} \wedge \theta_{i k} \wedge H_{j k}-\epsilon_{j} \theta_{i j} \wedge \theta_{j k} \wedge H_{i k}\right) \\
& =2 \sum_{i, j, k=1}^{n} \epsilon_{i} \theta_{i j} \wedge \theta_{i k} \wedge H_{j k}
\end{aligned}
$$

and, summarising,

$$
d \Psi=\frac{n(n-1) K}{2} \eta_{1} \wedge \cdots \wedge \eta_{n}-\frac{1}{2} \sum_{i, j, k=1}^{n} \epsilon_{i} \theta_{i j} \wedge \theta_{i k} \wedge H_{j k} .
$$

3.2. Sum of squares manifolds in different dimensions. We are now ready to give answers to the sum of squares problem on the given manifold $(\mathcal{M}, g)$. These answers depend on the dimension of $\mathcal{M}$. We have already dealt with the case where $n=1$ in Section 1.3. The two- and higher-dimensional cases differ significantly.

3.2.1. Two-dimensional sum of squares manifolds. In the case where $n=2$, the $(n-1)$-form $\Psi$ on $F(\mathcal{M})$ takes the simple form

$$
\Psi=\sum_{1 \leq i<j \leq 2} \theta_{i j} \wedge H_{i j}=\theta_{12}
$$

The last term in (3.8) vanishes here, so that

$$
d \Psi=K \eta_{1} \wedge \eta_{2}
$$

Therefore, by means of the independence condition (3.3), $d \Psi$ vanishes on any transverse two-dimensional submanifold of $F(\mathcal{M})$ if and only if $K \equiv 0$. Hence, if $(\mathcal{M}, g)$ is a (global or local) sum of squares manifold, it has to be flat because in dimension 2, scalar and Gaussian curvature coincide. On the other hand, any flat manifold is a local sum of squares manifold (see Section 1.4). Summarising the above, we obtain the following.

Theorem 3.2. A two-dimensional pseudo-Riemannian $C^{2}$-manifold is a local sum of squares manifold if and only if it is flat.

3.2.2. Higher-dimensional sum of squares manifolds. For dimensions larger than 2 there is no such restriction to be found, for we have the following.

Theorem 3.3. Any analytic pseudo-Riemannian manifold of dimension greater than two is a local sum of squares manifold.

Proof. By Proposition 3.1, we have to determine under which circumstances the $g$-orthonormal coframe bundle $F(\mathcal{M})$ of the manifold under consideration has an $n$-dimensional transverse integral manifold of the differential ideal $\mathcal{I}$ generated by $\Psi$ and $d \Psi$. We use the Cartan-Kähler theory as outlined in [3] to achieve this.

We begin with a characterisation of the transverse integral elements of $\mathcal{I}$. As is customary, we write $V_{n}\left(\mathcal{I}, \eta_{1} \wedge \cdots \wedge \eta_{n}\right)$ for the set of these elements of dimension $n$ 
(see [3], Chap. III, Def. 1.1). Since $\left\{\eta_{i}\right\}_{i=1, \ldots, n} \cup\left\{\theta_{i j}\right\}_{1 \leq i<j \leq n}$ defines a coframe on $F(\mathcal{M})$, the $n$-dimensional subspaces of the cotangent spaces to $F(\mathcal{M})$, in which the $\eta_{i}$ are independent, are described by equations of the form

$$
\theta_{i j}=\sum_{k=1}^{n} \gamma_{i j}^{k} \eta_{k} \quad \text { for all } 1 \leq i<j \leq n
$$

with some functions $\gamma_{i j}^{k}$ that satisfy

$$
\gamma_{i j}^{k}=-\gamma_{j i}^{k} \text { for all } i, j=1, \ldots, n
$$

(cf. [3], Chap. IV, Ex. 2.3). If, at a given point $\tilde{p}$ of $F(\mathcal{M})$, such a subspace is also dual to an integral element $E \subset T_{\tilde{p}} F(\mathcal{M})$ of $\mathcal{I}$, additional conditions have to hold for these coefficients. First, utilising (3.7), we obtain from the requirement that $\Psi$ vanishes on $E$ that

$$
\begin{aligned}
O & =\sum_{1 \leq i<j \leq n} \theta_{i j} \wedge H_{i j}=\sum_{1 \leq i<j \leq n} \sum_{k=1}^{n} \gamma_{i j}^{k} \eta_{k} \wedge H_{i j} \\
& =\sum_{i, j=1}^{n} \gamma_{i j}^{i}(-1)^{j} \eta_{1} \wedge \cdots \wedge \widehat{\eta_{j}} \wedge \cdots \wedge \eta_{n} \\
& =\sum_{j=1}^{n}\left(\sum_{i=1}^{n} \gamma_{i j}^{i}\right)(-1)^{j} \eta_{1} \wedge \cdots \wedge \widehat{\eta}_{j} \wedge \cdots \wedge \eta_{n} .
\end{aligned}
$$

In view of the independence of the $\eta_{i}$, the $\gamma_{i j}^{k}$ therefore also have to satisfy the $n$ equations

$$
\sum_{i=1}^{n} \gamma_{i j}^{i}=0 \quad \text { for all } j=1, \ldots, n .
$$

Analogously, the condition $d \Psi=O$ on $E$ yields from (3.8) that

$$
\begin{aligned}
\frac{n(n-1) K}{2} \eta_{1} \wedge \cdots \wedge \eta_{n} & =\frac{1}{2} \sum_{i, j, k=1}^{n} \epsilon_{i} \theta_{i j} \wedge \theta_{i k} \wedge H_{j k} \\
& =\frac{1}{2} \sum_{i, j, k, l, m=1}^{n} \epsilon_{i} \gamma_{i j}^{l} \eta_{l} \wedge \gamma_{i k}^{m} \eta_{m} \wedge H_{j k} \\
& =\frac{1}{2} \sum_{i, j, k, l, m=1}^{n} \epsilon_{i} \gamma_{i j}^{l} \gamma_{i k}^{m}\left(\delta_{l j} \delta_{m k}+\delta_{m j} \delta_{l k}\right) \eta_{1} \wedge \cdots \wedge \eta_{n}
\end{aligned}
$$

by (3.6). Therefore, taking (3.10) into consideration, we also demand that

$$
\sum_{i, j, k=1}^{n} \epsilon_{i} \gamma_{i j}^{k} \gamma_{i k}^{j}=n(n-1) K .
$$

Summarising, for any $n$-dimensional transverse integral element $E$ of $\mathcal{I}$ there exist $\gamma_{i j}^{k}$ satisfying (3.9)-(3.11) such that the annihilator of $E$ is spanned by the 1-forms

$$
\pi_{i j}:=\theta_{i j}-\sum_{k=1}^{n} \gamma_{i j}^{k} \eta_{k} \quad(1 \leq i<j \leq n)
$$


and vice versa. With these, the forms that generate $\mathcal{I}$ may be written as follows:

$$
\Psi=\sum_{1 \leq i<j \leq n} \pi_{i j} \wedge H_{i j}+\phi
$$

and

$$
\begin{aligned}
d \Psi= & \frac{n(n-1) K}{2} \eta_{1} \wedge \cdots \wedge \eta_{n} \\
& -\frac{1}{2} \sum_{i, j, k=1}^{n} \epsilon_{i}\left(\pi_{i j}+\sum_{l=1}^{n} \gamma_{i j}^{l} \eta_{l}\right) \wedge\left(\pi_{i k}+\sum_{m=1}^{n} \gamma_{i k}^{m} \eta_{m}\right) \wedge H_{j k} \\
= & -\frac{1}{2} \sum_{i, j, k, l=1}^{n} \epsilon_{i}\left(\gamma_{i j}^{l} \eta_{l} \wedge \pi_{i k} \wedge H_{j k}+\gamma_{i k}^{l} \pi_{i j} \wedge \eta_{l} \wedge H_{j k}\right)+\tilde{\phi} \\
= & -\sum_{i, j, k, l=1}^{n} \epsilon_{i} \gamma_{i k}^{l} \pi_{i j} \wedge \eta_{l} \wedge H_{j k}+\tilde{\phi} \\
= & \sum_{i, j, k=1}^{n} \epsilon_{i}(-1)^{k} \gamma_{i k}^{j} \pi_{i j} \wedge \eta_{1} \wedge \cdots \wedge \widehat{\eta_{k}} \wedge \cdots \wedge \eta_{n}+\tilde{\phi},
\end{aligned}
$$

where $\phi$ and $\tilde{\phi}$ are forms of degree 2 or more in the $\pi_{i j}$ or vanish on $E$ by construction. These representations now may be used to determine the Cartan characters for $E$. Following the procedure outlined in [3], Chap. III, Prop. 1.15, we consider the integral flag $O \subset E_{1} \subset \cdots \subset E_{n}=E$, where for each $l<n$ the $l$-dimensional integral element $E_{l}$ is given by

$$
E_{l}:=\left\{v \in T_{\tilde{p}} F(\mathcal{M}) \mid \eta_{i}(v)=0 \quad \text { for all } i>l\right\} .
$$

We first calculate the codimensions $c_{l}$ of the polar spaces $H\left(E_{l}\right)$ ([3], Chap. III, Def. 1.5) of these integral elements. For this the following terminology is used: if $\rho:=\left(\rho_{1}, \ldots, \rho_{k}\right)$ is a multi-index of degree $l$ taken from the set $\{1, \ldots, n\}$ (in short: $\rho \in \mathbb{N}_{n}^{l}$ ), we write $\sup \rho$ for the largest of the integers in $\left\{\rho_{1}, \ldots, \rho_{l}\right\}$. We now define

$$
\pi_{\left(\rho_{1}, \ldots, \rho_{n-2}\right)}:= \begin{cases}(-1)^{i+j-1} \pi_{i j} & \text { if } \rho_{1}<\cdots<\rho_{n-2} \text { and } i<j \text { are such } \\ O & \text { that }\left\{\rho_{1}, \ldots, \rho_{n-2}, i, j\right\}=\{1, \ldots, n\},\end{cases}
$$

respectively

$$
\tilde{\pi}_{\left(\rho_{1}, \cdots, \rho_{n-1}\right)}:= \begin{cases}\sum_{i, j,=1}^{n} \epsilon_{i}(-1)^{k} \gamma_{i k}^{j} \pi_{i j} & \text { if } \rho_{1}<\cdots<\rho_{n-1} \text { and } k \text { are such } \\ O & \text { that }\left\{\rho_{1}, \ldots, \rho_{n-1}, k\right\}=\{1, \ldots, n\} \\ \text { otherwise. }\end{cases}
$$

With these, (3.12) and (3.13) take the simpler forms

$$
\Psi=\sum_{\rho \in \mathbb{N}_{n}^{n-2}} \pi_{\rho} \wedge \eta_{\rho_{1}} \wedge \cdots \wedge \eta_{\rho_{n-2}}+\phi
$$

and

$$
d \Psi=\sum_{\rho \in \mathbb{N}_{n}^{n-1}} \tilde{\pi}_{\rho} \wedge \eta_{\rho_{1}} \wedge \cdots \wedge \eta_{\rho_{n-1}}+\tilde{\phi}
$$


Then, by [3], Chap. III, Prop. 1.15, for any $l=0, \ldots, n-1, c_{l}$ is the number of independent 1 -forms in the set

$$
\mathcal{L}_{l}:=\left\{\pi_{\rho}, \tilde{\pi}_{\rho} \mid \sup \rho \leq l\right\} .
$$

Since the multi-indices under consideration here are at least of degree $n-2$, we have

$$
c_{l}=0 \text { for all } l=0, \ldots, n-3 .
$$

In the case where $l=n-2, \mathcal{L}_{n-2}$ contains only one nonzero element, namely $\pi_{(1, \ldots, n-2)}=\pi_{n-1, n}$, and thus

$$
c_{n-2}=1 .
$$

In order to determine $c_{n-1}$, we have to evaluate the number of linearly independent 1 -forms in

$$
\begin{aligned}
\mathcal{L}_{n-1} & =\left\{\pi_{(1, \ldots, \hat{i}, \ldots, n-1)}, \tilde{\pi}_{(1, \ldots, n-1)} \mid i=1, \ldots, n-1\right\} \\
& =\left\{(-1)^{n+i-1} \pi_{i n} \mid i=1, \ldots, n-1\right\} \cup\left\{\sum_{i, j=1}^{n} \epsilon_{i}(-1)^{n} \gamma_{i n}^{j} \pi_{i j}\right\} .
\end{aligned}
$$

Hence

$$
c_{n-1}= \begin{cases}n & \text { if there exist } i<j<n \text { with } \gamma_{n-1, i}^{j} \neq 0, \\ n-1 & \text { otherwise. }\end{cases}
$$

We return to the analysis of the coefficients associated with the $n$-dimensional transverse integral elements of $\mathcal{I}$. From the above, we know that the elements in $V_{n}\left(\mathcal{I}, \eta_{1} \wedge \cdots \wedge \eta_{n}\right)$ are characterised by $\gamma_{i j}^{k}$ which satisfy the relations (3.9)-(3.11). In other words, these coefficients form those vectors in the linear space

$$
\Gamma:=\left\{\left(\gamma_{i j}^{k}\right) \mid \gamma_{i j}^{k}=-\gamma_{j i}^{k} \text { and } \sum_{i=1}^{n} \gamma_{i j}^{i}=0 \quad \text { for all } i, j, k=1, \ldots, n\right\}
$$

that satisfy (3.11), that is, they form the contour hypersurface

$$
\Gamma_{K}:=\left\{\left(\gamma_{i j}^{k}\right) \in \Gamma \mid \sum_{i, j, k=1}^{n} \epsilon_{i} \gamma_{i j}^{k} \gamma_{i k}^{j}=n(n-1) K\right\} .
$$

Since $n>3$, it follows that the gradient of the mapping

$$
q: \Gamma \rightarrow \mathbb{R}:\left(\gamma_{i j}^{k}\right) \mapsto \sum_{i, j, k=1}^{n} \epsilon_{i} \gamma_{i j}^{k} \gamma_{i k}^{j}
$$

vanishes if and only if $\left(\gamma_{i j}^{k}\right)=O$. As a consequence, this mapping is a nondegenerate quadratic form. We denote the associated bilinear form by

$$
s(\gamma, \eta):=\frac{1}{2}(q(\gamma+\eta)-q(\gamma)-q(\eta)) \quad \text { for all } \gamma, \eta \in \Gamma
$$

and let

$$
\Gamma^{ \pm}:=\left\{\left(\gamma_{i j}^{k}\right) \in \Gamma \mid \pm q\left(\gamma_{i j}^{k}\right) \geq 0\right\} .
$$

For any choice of $\gamma, \eta \in \Gamma^{+}$we obtain that

$$
q(\gamma+\eta)=q(\gamma)+q(\eta)-2 s(\gamma, \eta) \geq q(\gamma)+q(\eta)-2 \sqrt{q(\gamma) q(\eta)} \geq 0
$$


from the Cauchy-Schwarz inequality. Moreover, scalar multiplication of the argument of $q$ does not change its sign. Hence, $\Gamma^{+}$is a linear subspace of $\Gamma$. Similar arguments yield the same property for $\Gamma^{-}$. Since $q$ is nondegenerate, this implies that we may write $\Gamma$ as the direct sum of $\Gamma^{+}$and $\Gamma^{-}: \Gamma=\Gamma^{+} \oplus \Gamma^{-}$. It is also easily seen that both vector spaces $\Gamma^{+}$and $\Gamma^{-}$have positive dimension. Hence $q$ is indefinite on $\Gamma$ and therefore maps onto all of $\mathbb{R}$. Thus, by the implicit function theorem, $\Gamma_{K}$ defines a smooth submanifold of $\Gamma$ unless $K=0$. In that case, the only singular point of the hypersurface $\Gamma_{0}$ is at $\gamma_{i j}^{k}=0$ for all $i, j, k=1, \ldots, n$. Consequently, all $n$-dimensional transverse integral elements of $\mathcal{I}$, except for the one associated with $\left(\gamma_{i j}^{k}\right)=O$, admit smooth submanifolds of $V_{n}\left(\mathcal{I}, \eta_{1} \wedge \cdots \wedge \eta_{n}\right)$ which pass through it. The dimension of these manifolds is

$$
\operatorname{dim} \Gamma-1=N n-n-1=\frac{n^{2}(n-1)}{2}-n-1 .
$$

Observe that this number is greater than 4 for $n \geq 3$.

It is easy to find $\gamma_{i j}^{k}$ with (3.9)-(3.11) such that $c_{n-1}=n$. We concentrate on these cases, where we have

$$
c_{l}= \begin{cases}0 & \text { if } l=0, \ldots, n-3 \\ 1 & \text { if } l=n-2 \\ n & \text { if } l=n-1\end{cases}
$$

The Cartan characters are now defined by $s_{0}:=c_{0}, s_{l}:=c_{l-1}-c_{l}$ for $l=$ $1, \ldots, n-1$, so that

$$
s_{l}= \begin{cases}0 & \text { if } l=0, \ldots, n-3, \\ 1 & \text { if } l=n-2, \\ n-1 & \text { if } l=n-1,\end{cases}
$$

here. Defining the $n$-th pseudocharacter $s_{n}$ by the identity

$$
s_{n}:=\operatorname{dim} F(\mathcal{M})-n-c_{n-1}=\frac{n(n-1)}{2}-n=\frac{n(n-3)}{2}
$$

(see [3], Chap. III, Prop. 2.4), we obtain that

$$
\sum_{l=1}^{n-1} l s_{l}=(n-2)+(n-1)(n-1)+n \frac{n(n-3)}{2}=\frac{n^{2}(n-1)}{2}-n-1 .
$$

This is the same value as was calculated in (3.14) for the dimension of the space of transverse integral elements for $\mathcal{I}$ over a point in $F(\mathcal{M})$. Now, by Cartan's test ([3], Chap. III, Theorem 1.11), and our above discussion on the smoothness of the $\Gamma_{K}$, we have that the integral elements $E_{l}$ associated with the chosen $\gamma_{i j}^{k}$ are regular for all $l<n$, in particular implying that $E_{n}$ is ordinary. In other words, the differential ideal $\mathcal{I}$ with independence condition (3.3) is in involution.

Since we have assumed analyticity, we may now use the Cartan-Kähler theorem. It states ([3], Chap. III, Cor. 2.3) that under the given circumstances any ordinary integral element can be integrated to a submanifold which is an integral manifold of $\mathcal{I}$ by construction. Proposition 3.1 then implies the assertion of the theorem.

Remark 3.4. The Cartan-Kähler theorem also provides some information on the size of the solution space of the sum of squares problem. This is because the integral submanifold that has been constructed depends on $s_{n}$ arbitrary (up to specification of the initial conditions) analytic functions of $n$ variables (cf. [16], Theorem 15.7). 


\section{Applications}

In this section we briefly outline some applications of the results established in the above. The Laplace-Beltrami operator appears in each of the main classical linear second-order partial differential equations, namely the Laplace, heat, and wave equations. We concentrate on investigating these and some related topics.

Given a list of vector fields $X_{1}, \ldots, X_{k}$ on an open subset $\mathcal{U}$ of an $n$-dimensional differential manifold which satisfy Hörmander's condition (that is, they generate $\mathfrak{X}(\mathcal{U})$ by the Lie bracket operation), one defines the associated Laplacian of these vector fields as the operator

$$
\sum_{i=1}^{k} X_{i}^{2} .
$$

Such operators, sometimes also called "sub-Laplacians", which are hypoelliptic by a famous result of Hörmander (see [12]), have been the object of numerous investigations. The main objective in the literature on Hörmander associated Laplacians has been to understand (4.1) as a nonelliptic operator and derive some of its properties. However, as we have shown in the previous sections, in the case where $k=n$ on certain manifolds and for certain choices of vector fields in (4.1), this operator is the usual Laplace-Beltrami operator and consequently elliptic. In the following we use our results to draw some connections between the work that has been done on Hörmander associated Laplacians and the Laplace-Beltrami operator.

4.1. Lie groups. Sub-Laplacians have been studied in particular on Lie groups. Because of this, most of the applications of our results evolve in the interface between general Riemannian manifolds and Lie groups.

Any Lie group $(\mathcal{G}, \star)$ is an analytic manifold that can be turned into a Riemannian manifold in a natural way, i. e., by adapting a positive definite bilinear form $Q$ on its Lie algebra $\mathfrak{G}$. Identifying the elements of $\mathfrak{G}$ with the left-invariant vector fields on $\mathcal{G}$, there is a unique Riemannian metric with $Q$ on $T_{e} \mathcal{G} \cong \mathfrak{G}$, and it is leftinvariant. The Riemannian manifold $\left(\mathcal{G}, g_{Q}\right)$ is usually called $\mathcal{G}$ with a left-invariant metric.

It is well-known that any unimodular Lie group $(\mathcal{G}, \star)$ (endowed with such a metric) has the sum of squares property, even globally. This is because then any left-invariant vector field has divergence zero ([22], Prop. A1.1). Now, from Theorem 3.3, we get immediately that any Lie group of dimension greater than two has the local sum of squares property, no matter what Riemannian metric is introduced on the manifold. In the one-dimensional (trivial) case this holds, too, as we have seen in Section 1.3. For $n=2$ there are only two connected simply connected Lie groups to consider. One is $\mathbb{R}^{2}$ (which is clearly flat with respect to the metric induced by the Euclidean inner product on $\mathfrak{G})$ and the other one is the $(a x+b)$-group ([16], Ex. 2.11), which is isomorphic to the two-dimensional hyperbolic space. This Riemannian manifold has Gaussian curvature $\kappa=-1$, and thus, by Theorem 3.2, there do not exist vector fields on $\left(\mathbb{R}^{+} \times \mathbb{R}, g\right)$ such that the Laplacian equals the sum of their squares, even locally. Hence there is exactly one connected simply connected Lie group endowed with a left-invariant metric that is not a local sum of squares manifold. 
Theorem 4.1. Let $(\mathcal{G}, \star)$ be a Lie group endowed with the left-invariant metric $g$ induced by the Euclidean inner product on its Lie algebra. Then $(\mathcal{G}, g)$ is a local sum of squares manifold if and only if $(\mathcal{G}, \star)$ is not isomorphic to the $(a x+b)$-group.

Remark 4.2. Note that the sum of squares vector fields in the theorem are not necessarily left-invariant with respect to the group action (which can be achieved in the unimodular case).

4.2. Heat kernels. Consider the general heat equation

$$
\left(\frac{\partial}{\partial t}-\mathfrak{L}\right) u=0
$$

with $\mathfrak{L}$ a second-order differential operator acting on a Riemannian manifold. Selecting the Laplace-Beltrami operator $\Delta$ for $\mathfrak{L}$ in (4.2) yields the usual heat equation, which has been investigated, for instance, in [21]. Other authors, however, choose $\mathfrak{L}$ as a sum of squares of vector fields or consider more general hypoelliptic operators.

One main objective in the analysis of this equation is to determine its fundamental solution, the heat kernel. In Euclidean space and for $\mathfrak{L}=\Delta$ it is given by

$$
H_{t}(x, y)=\frac{e^{-(x-y)^{2} / 4 t}}{(4 \pi t)^{n / 2}} .
$$

On arbitrary Riemannian manifolds and/or for other $\mathfrak{L}$ such an explicit formula for the associated heat kernel is very hard to find. In order to obtain some information on the solutions to the heat equation, for example about their regularity or asymptotic behaviour, one may nevertheless use estimates for this kernel.

Interestingly, much of the work in this connection has been carried out not for the Laplace-Beltrami operator but for Hörmander associated Laplacians. This is mainly due to the fact that the latter are easier to handle than arbitrary hypoelliptic operators, because one does not require a deep result of Rothschild and Stein ([20]) to control the first-order terms in such an operator. See, for instance, [23], Chapter V, for these more economical proofs of heat kernel estimates.

On sum of squares manifolds this problem can be avoided for the original heat equation $(\mathfrak{L}=\Delta)$ by considering a set of sum of squares vector fields (which satisfies Hörmander's condition by Lemma 2.1). This then yields an easier and direct way to obtain such estimates on Riemannian manifolds.

\subsection{Brownian motion. Let}

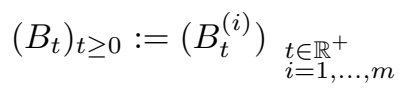

be a Brownian motion on $\mathbb{R}^{m}$, i. e., each component $\left(B_{t}^{(i)}\right)_{t \in \mathbb{R}^{+}}$is an independent almost-surely continuous stochastic process with independent increments such that, for each $t$, the random variable $B_{t}^{(i)}$ is centred, Gaussian, and has variance $t$.

There are two common approaches to defining Brownian motion also on Riemannian manifolds. In both cases, this stochastic process is defined as a solution to a stochastic differential equation. The characteristic feature of such a process is that its generator is $\frac{1}{2} \Delta$ (see [7] for details).

The first - and most widely used - way to define a Brownian motion on a Riemannian manifold is the method of "rolling without slipping" the manifold along 
a Brownian path in $\mathbb{R}^{n}$. One obtains the process as a solution to a Stratonovich equation involving horizontal vector fields on the $g$-orthonormal frame bundle ([13], Chap. V).

On Lie groups the common approach to defining a Brownian motion $\left(\xi_{t}\right)_{t \geq 0}$ starting at $p$ is by selecting an orthonormal basis on its Lie algebra, considering the corresponding left-invariant vector fields $\left(X_{1}, \cdots, X_{n}\right)$, and seeking the solution of the following stochastic differential equation ([22], Sec. 4):

$$
d \xi_{t}=\left.\sum_{i=1}^{n} X_{i}\right|_{\xi_{t}}{ }^{\circ} d B_{t}^{(i)} \text { with } \xi_{0}=p
$$

which is another way of writing the integral equation

$$
f\left(\xi_{t}\right)=f(p)+\sum_{i=1}^{m} \int_{0}^{t}\left(X_{i} f\right)\left(\xi_{s}\right){ }^{\circ} d B_{s}^{(i)} .
$$

Here ${ }^{\circ} d$ denotes the Stratonovich differential. This construction is called the direct approach, because it does not require one to go up to the $g$-orthonormal frame bundle. We may generalise (4.3) to Riemannian manifolds by simply demanding that $\left(X_{i}\right)_{i}$ is an arbitrary $g$-orthonormal frame there. Converting Stratonovich to Itô integrals in the corresponding integral equation yields that

$$
f\left(\xi_{t}\right)=f(p)+\sum_{i=1}^{m} \int_{0}^{t}\left(X_{i} f\right)\left(\xi_{s}\right) d B_{s}^{(i)}+\frac{1}{2} \sum_{i=1}^{m} \int_{0}^{t}\left(X_{i}^{2} f\right)\left(\xi_{s}\right) d s,
$$

implying that the generator here is given by one half the sum of squares of the vector fields $X_{i}$.

The main difference between the two approaches sketched above thus lies in their generators. The horizontal construction ensures that one half the Laplacian is, as desired, the generator of the Brownian motion, which is not necessarily true for the direct approach. However, the latter leaves a much wider freedom of choice in the construction because it permits the use of an arbitrary $g$-orthonormal frame in (4.3). On a sum of squares manifold, we can thus use a list of sum of squares vector fields $\left(X_{i}\right)_{i}$ such that the last term in (4.4) equals $\frac{1}{2} \int_{0}^{t} \Delta f\left(\xi_{s}\right) d s$. Then, the direct approach also yields a stochastic process with one half the Laplace-Beltrami operator as generator, and we are justified in calling this process a Brownian motion. Our results given in Sections 1.3, 1.4, Theorem 3.2, and Theorem 3.3 thus imply the following.

Theorem 4.3. Let $(\mathcal{M}, g)$ be a differentiable Riemannian manifold of dimension $n$ which is in addition

(i) flat if $n=2$.

(ii) analytic if $n \geq 3$.

Then one can find locally a list of vector fields $\left(X_{i}\right)_{i}$ such that the process defined by the direct Stratonovich equation (4.3) is a Brownian motion on $(\mathcal{M}, g)$, i. e., a stochastic process with one half the Laplace-Beltrami operator as generator.

When the manifold is two-dimensional and nonflat, this is not possible.

For a closing remark we return to the Lie group setting. It is well known that the direct approach defines a Brownian motion on unimodular Lie groups (cf. Section 4.1). The deeper reason for this lies in the fact that left translation on such 
manifolds is divergence-free, which is not true in general. However, combining our Theorems 4.1 and 4.3, we see that the only Lie group to be excluded here is the $(a x+b)$-group.

Corollary 4.4. Let $(\mathcal{G}, \star)$ be any Lie group endowed with the left-invariant metric $g$ induced by the Euclidean inner product on its Lie algebra. Assume that $(\mathcal{G}, g)$ is not isomorphic to the $(a x+b)$-group.

Then one can find locally a list of vector fields $\left(X_{i}\right)_{i}$ on $\mathcal{G}$ such that the process defined by the direct Stratonovich equation (4.3) is a Brownian motion on $(\mathcal{G}, \star)$, $i$. e., a stochastic process with one half the Laplace-Beltrami operator as generator.

When $(\mathcal{G}, g)$ is isomorphic to the $(a x+b)$-group, this cannot be achieved.

4.4. Waves. The wave operator on any pseudo-Riemannian manifold $(\mathcal{M}, g)$ coincides with the Laplacian on the manifold $\mathbb{R}^{+} \times \mathcal{M}$ with a pseudo-Riemannian metric given by

$$
-\langle\cdot, \cdot\rangle \otimes g(\cdot, \cdot) \quad \text { acting on }\left(\mathfrak{X}\left(\mathbb{R}^{+}\right) \otimes \mathfrak{X}(\mathcal{M})\right) \times\left(\mathfrak{X}\left(\mathbb{R}^{+}\right) \otimes \mathfrak{X}(\mathcal{M})\right) .
$$

With this in mind, we may combine the analysis of the Laplace and the wave equation on these manifolds here.

Pseudo-Riemannian manifolds of index 1 are commonly called Lorentz manifolds. As we just have mentioned, their Laplacian is the general wave operator $\square$, also known as the d'Alembert operator. On any $n$-dimensional Lorentzian sum of squares manifold $(n \geq 2)$ we thus have

$$
\square=-T^{2}+\sum_{i=1}^{n-1} X_{i}^{2}
$$

for a list $\left(T, X_{1}, \ldots, X_{n-1}\right)$ of sum of squares vector fields. From Lemma 2.1, we know that such a list is $g$-orthonormal, and in view of the given signature we have in particular

$$
g(T, T)=-1 \quad \text { and } \quad g\left(X_{i}, X_{i}\right)=1 \quad \text { for all } i=1, \ldots, n-1 .
$$

Therefore, by definition, the vector field $T$ is timelike, whereas the $X_{i}$ are spacelike. Consequently, the wave equation $\square u=f$, when expressed with respect to sum of squares vector fields, on such a manifold takes precisely the same form as on $\mathbb{R}^{+} \times \mathbb{R}^{n-1}$. However, in contrast with the Euclidean case, these vector fields generally do not commute. Indeed, by Corollary 2.5 , they can commute only on flat Lorentzian manifolds. Hence on curved Lorentzian manifolds there is no coordinate system such that the wave equation takes exactly the Euclidean form.

Relativity. In any case, the d'Alembertian on a Lorentzian sum of squares manifold can be written in the form (4.5). From Theorem 3.3 we know that for $n \geq 3$ this can locally always be achieved, provided the manifold is analytic, no matter what (analytic) Lorentzian metric has been imposed on it. In particular, this applies to the case where $n=4$ and thus to General Relativity. There the main objective is to find metrics that satisfy Einstein's field equations and subsequently solve the wave equation on the resulting manifold (see [10]). Therefore, wherever a solution of the field equation is analytic, the d'Alembertian may be written in the familiar form (4.5).

In Special Relativity (and only there), where flat universes are considered, the vector fields involved even form an integrable system, because of the Frobenius theorem and the reasons given above. 
Finite propagation speed. The linear wave equation on Riemannian manifolds has been extensively studied in the past decades. Results on existence and uniqueness have been established locally as well as globally on specific Riemannian manifolds (see, e. g., [9] or [2]). The property of finite propagation speed for these solutions has been proved along the way and was further used to establish estimates for functions of the Laplace-Beltrami operator, for example in [5]. A study of these results leads to the impression that in order to establish the main features of solutions to the wave equation, a sum of squares structure of the Laplacian is not particularly important. The symmetry of this operator on $L^{2}(\mathcal{M})$ given by its "div grad" structure seems to be of far more significance here. This feature is opposed to the heat equation, where a sum of squares property of the Laplacian is helpful to obtain information on its solutions.

\section{REFERENCES}

1. R. Abraham, J.E. Marsden, and T. Ratiu, Manifolds, Tensor Analysis, and Applications, Springer, Berlin, 1988. MR 89f:58001

2. P.H. Bérard, On the wave equation on a compact Riemannian manifold without conjugate points, Math. Z. 155 (1977), 249-276. MR 56:13295

3. R.L. Bryant, S.S. Chern, R.B. Gardner, H.L. Goldschmidt, and P.A. Griffiths, Exterior Differential Systems, Springer, Berlin, 1991. MR 92h:58007

4. I. Chavel, Riemannian Geometry: a Modern Introduction, Cambridge University Press, Cambridge, 1993. MR 95j:53001

5. J. Cheeger, M. Gromov, and M. Taylor, Finite propagation speed, kernel estimates for functions of the Laplace operator, and the geometry of complete Riemannian manifolds, J. Differ. Geom. 17 (1982), 15-53. MR 84b:58109

6. L. Conlon, Differentiable Manifolds. A First Course, Birkhäuser, Basel, 1993. MR 94d:58001

7. M.J. Cornwall, Brownian Motion and Heat Kernels on Lie Groups, Ph.D. thesis, University of New South Wales, Sydney, 1994.

8. G. de Rham, Differentiable Manifolds, Springer, Berlin, 1984. MR 85m:58005

9. F.G. Friedlander, The Wave Equation on a Curved Space-time, Cambridge University Press, Cambridge, 1975. MR 57:889

10. S.W. Hawking and G.F.R. Ellis, The Large Scale Structure of Space-time, Cambridge University Press, Cambridge, 1973. MR 54:12154

11. S. Helgason, Differential Geometry, Lie Groups, and Symmetric Spaces, Academic Press, New York, 1978. MR 80k:53081

12. L. Hörmander, Hypoelliptic second order differential equations, Acta Math. 119 (1968), 147171. MR 36:5526

13. N. Ikeda and S. Watanabe, Stochastic Differential Equations and Diffusion Processes, 2nd ed., North-Holland, Amsterdam, 1989. MR 90m:60069

14. S. Lang, Differential and Riemannian Manifolds, Springer, Berlin, 1995. MR 96d:53001

15. D. Martin, Manifold Theory: an Introduction for Mathematical Physicists, E. Horwood, New York, 1991. MR 92g:58001

16. P.J. Olver, Equivalence, Invariants, and Symmetry, Cambridge University Press, Cambridge, 1995. MR 96i: 58005

17. B. O'Neill, Semi-Riemannian Geometry: with Applications to Relativity, Academic Press, New York, 1983. MR 85f:53002

18. W.H. Paus, Sum of squares manifolds: The expressibility of the Laplace-Beltrami operator on pseudo-Riemannian manifolds as a sum of squares of vector fields, Ph.D. thesis, University of New South Wales, Sydney, 1996.

19. J.F. Ritt, Differential Algebra, Dover, New York, 1966. MR 34:1315

20. L.P. Rothschild and E.M. Stein, Hypoelliptic differential operators and nilpotent Lie groups, Acta Math. 137 (1977), 247-320. MR 55:9771

21. R.S. Strichartz, Analysis of the Laplacian on the complete Riemannian manifold, J. Funct. Anal. 52 (1983), 48-79. MR 84m:58138 
22. J.C. Taylor, The Iwasawa decomposition and the limiting behaviour of Brownian motion on a symmetric space of non-compact type, Contemp. Math. 73 (1988), 303-331. MR 89f:58139

23. N. Varopoulos, L. Saloff-Coste, and T. Coulhon, Analysis and Geometry of Groups, Cambridge University Press, Cambridge, 1992. MR 95f:43008

24. J.A. Wolf, Spaces of Constant Curvature, 3rd ed., Publish or Perish, Boston, 1974. MR 49:7958

25. H.-H. Wu, The Bochner technique in differential geometry, Math. Rep. 3 (1988), 289-538. MR 91h:58031

School of Mathematics, The University of New South Wales, Sydney NSW 2052, Australia

Current address: Deutsche Bank AG, Credit Risk Management, 60262 Frankfurt am Main, Germany

E-mail address: wilfried.paus@zentrale.deuba.com 